\title{
Constructive Diophantine approximation in generalized continued fraction Cantor sets
}

by

Kalle LeppäLÄ (Aarhus) and Topi Törmä (Oulu)

1. Introduction. By an irrationality exponent of a real number $\tau$ we mean any exponent $\mu$ for which there exist positive constants $c$ and $q_{0}$ such that the inequality

$$
\left|\tau-\frac{p}{q}\right| \geq \frac{c}{q^{\mu}}
$$

holds for all $p, q \in \mathbb{Z}, q \geq q_{0}$. The asymptotic irrationality exponent $\mu_{I}(\tau)$ is then defined as the infimum of all such exponents $\mu$, with the convention that the infimum of the empty set is $\infty$. If $\mu_{I}(\tau)=\infty$, then $\tau$ is called a Liouville number, and if $\mu_{I}(\tau)>2$, it is called very well approximable.

The set of all possible asymptotic irrationality exponents is

$$
\left\{\mu_{I}(\tau): \tau \in \mathbb{R}\right\}=\{1\} \cup[2, \infty] .
$$

It is an easy application of the triangle inequality and $b$-ary expansion of $\tau$ to see that $\mu_{I}(\tau)=1$ for $\tau \in \mathbb{Q}$. For $\tau \in \mathbb{R} \backslash \mathbb{Q}$ we have $\mu_{I}(\tau) \geq 2$ by Dirichlet's theorem on Diophantine approximation $[\underline{S}$. One way to see that all the values in $[2, \infty]$ actually occur is constructing explicit examples using the theory of simple continued fractions and Lemma 2.1. In the metrical sense the typical asymptotic irrationality exponent is 2: by Khinchin's theorem [S] the set of very well approximable numbers has Lebesgue measure zero.

Which values of $\mu_{I}(\tau)$ are possible if we replace $\mathbb{R}$ with some interesting smaller set?

Mahler [M] asked this question for the middle-third Cantor set

$$
C=\left\{\sum_{n=1}^{\infty} \frac{c_{n}}{3^{n}}: c_{n} \in\{0,2\} \text { for all } n\right\} .
$$

2010 Mathematics Subject Classification: Primary 11J82, 11J70; Secondary 11K50.

Key words and phrases: irrationality exponent, generalized continued fraction, metric theory of continued fractions.

Received 8 January 2018; revised 17 July 2018.

Published online *. 
All the exponents $\mu_{I}(\tau) \in\{1\} \cup[2, \infty]$ are still possible, as was shown by Levesley, Salp and Velani [LSV] for $\mu_{I}(\tau) \in\{1\} \cup((3+\sqrt{5}) / 2, \infty]$ and Bugeaud [B] for the trickier part $\mu_{I}(\tau) \in[2,(3+\sqrt{5}) / 2]$, using the folding lemma.

We ask the question on the generalized continued fraction Cantor sets

$$
E_{\mathcal{B}}^{\mathcal{A}}=\left\{\frac{a_{1}}{b_{1}+\frac{a_{2}}{b_{2}+\cdots}}: a_{n} \in \mathcal{A}, b_{n} \in \mathcal{B} \text { for all } n\right\},
$$

where $\mathcal{A}$ and $\mathcal{B}$ are some given finite sets of positive integers. In [HLMT] we took the first steps in studying this problem and saw that not everything is always possible. We proved:

TheOREM 1.1. Let $\alpha_{1}$ and $\alpha_{2}$ be the least and greatest elements of $\mathcal{A}$, respectively, and $\beta_{1}$ the least element of $\mathcal{B}$. If $\alpha_{2} \leq \beta_{1}$, then

$$
\mu_{I}(\tau) \leq 2+\frac{\log \alpha_{2}}{\log \left(\beta_{1}+\sqrt{\beta_{1}^{2}+4 \alpha_{1}}\right)-\log \left(2 \alpha_{2}\right)}
$$

for all $\tau \in E_{\mathcal{B}}^{\mathcal{A}}$.

In fact, in [HLMT] we gave a bit more complicated condition which turns out to be equivalent to $\alpha_{2} \leq \beta_{1}$.

At this point it still was thinkable that the generalized continued fraction Cantor sets only contain elements with the metrically typical asymptotic irrationality exponent 2 , as is the case with the simple continued fraction Cantor sets (i.e. the case $\mathcal{A}=\{1\}$ ), which only contain badly approximable numbers [S. However, in HLMT we also constructed explicit examples $\tau \in E_{\{1\}}^{\{1,2\}}$ with any prescribed $\mu_{I}(\tau) \in\{1\} \cup[2, \infty]$. In the present work we will prove a more general result giving sufficient conditions under which the set $E_{\mathcal{B}}^{\mathcal{A}}$ contains elements with any prescribed asymptotic irrationality exponent. Our method is inspired by Raney's algorithm on linear fractional transformations of simple continued fractions $[\mathrm{R}]$. Furthermore, we show that there exist sets with this property and arbitrarily small Hausdorff dimension.

One might speculate that perhaps the generalized continued fraction Cantor sets fall into two categories: wild Cantor sets like the case $\mathcal{A}=\{1,2\}$, $\mathcal{B}=\{1\}$, containing elements with any prescribed asymptotic irrationality exponent from $\{1\} \cup[2, \infty]$, and non-wild Cantor sets, like the simple continued fraction Cantor sets, which only contain elements with the metrically typical asymptotic irrationality exponent 2 . In this work, we show that the answer is more complicated than that: a generalized continued fraction Cantor set might contain very well approximable numbers even if it does not contain Liouville numbers. 
2. Basics about continued fractions. The generalized continued fraction

$$
\tau=b_{0}+\frac{a_{1}}{b_{1}+\frac{a_{2}}{b_{2}+\cdots}}=b_{0}+\frac{a_{1}}{b_{1}}+\frac{a_{2}}{b_{2}}+\cdots
$$

is defined as the limit of the $n$th convergent

$$
b_{0}+\frac{a_{1}}{b_{1}+\frac{a_{2}}{b_{2}+\cdots+\frac{a_{n}}{b_{n}}}}=b_{0}+\frac{a_{1}}{b_{1}}+\frac{a_{2}}{b_{2}}+\cdots+\frac{a_{n}}{b_{n}}
$$

as $n$ tends to infinity. This limit does not necessarily exist, but it does when $a_{n}$ and $b_{n}$ are positive bounded integers.

The numerators $A_{n}$ and the denominators $B_{n}$ of the $n$th convergent can be calculated with the recurrence formulae

$$
\left\{\begin{array} { l } 
{ A _ { n + 1 } = b _ { n + 1 } A _ { n } + a _ { n + 1 } A _ { n - 1 } , } \\
{ B _ { n + 1 } = b _ { n + 1 } B _ { n } + a _ { n + 1 } B _ { n - 1 } , }
\end{array} \quad \left\{\begin{array} { l } 
{ A _ { 0 } = b _ { 0 } , } \\
{ B _ { 0 } = 1 , }
\end{array} \quad \left\{\begin{array}{l}
A_{1}=b_{0} b_{1}+a_{1}, \\
B_{1}=b_{1} .
\end{array}\right.\right.\right.
$$

The numbers $A_{n}$ and $B_{n}$ might have a non-trivial common factor, with size bounded by $a_{1} \cdots a_{n}$ as can be seen from the determinant formula

$$
A_{n+1} B_{n}-A_{n} B_{n+1}=(-1)^{n} \prod_{k=1}^{n+1} a_{k} .
$$

When $a_{n}=1$ for all $n$, the continued fraction is called simple. Every irrational number has a unique simple continued fraction expansion. For clarity, we will use different letters $b_{n}=d_{n}, A_{n}=C_{n}$ and $B_{n}=D_{n}$ to indicate when we are talking about the simple expansion. By (2.1), $C_{n}$ and $D_{n}$ are coprime, and telescoping gives

$$
\frac{C_{n}}{D_{n}}-d_{0}=\sum_{k=0}^{n-1}\left(\frac{C_{k+1}}{D_{k+1}}-\frac{C_{k}}{D_{k}}\right)=\sum_{k=0}^{n-1} \frac{(-1)^{k}}{D_{k} D_{k+1}},
$$

so

$$
\frac{C_{n}}{D_{n}}=d_{0}+\sum_{k=0}^{n-1} \frac{(-1)^{k}}{D_{k} D_{k+1}} \quad \text { and } \quad \tau=d_{0}+\sum_{k=0}^{\infty} \frac{(-1)^{k}}{D_{k} D_{k+1}} .
$$

The usual error estimate of an alternating series together with the recursion formulae now give

$$
\frac{1}{\left(d_{n+1}+2\right) D_{n}^{2}}<\left|\tau-\frac{C_{n}}{D_{n}}\right|<\frac{1}{d_{n+1} D_{n}^{2}} .
$$

On the other hand, it can be shown [S] that if $|\tau-p / q|<1 /\left(2 q^{2}\right)$, then $p / q$ must be a convergent of the simple continued fraction expansion of $\tau$. Combined, these facts give a formula for calculating the asymptotic irrationality exponent: 
Lemma 2.1. Let

$$
\tau=d_{0}+\frac{1}{d_{1}}+\frac{1}{d_{2}}+\cdots
$$

Then

$$
\mu_{I}(\tau)=2+\limsup _{n \rightarrow \infty} \frac{\log d_{n+1}}{\log D_{n}} .
$$

To obtain the asymptotic irrationality exponent of a number $\tau$, we ideally need the simple continued fraction expansion of $\tau$ in order to use Lemma2.1. Our strategy is to transform the generalized continued fraction expansion into a simple one with infinitely many applications of Raney's algorithm $[\mathbb{R}$ on linear fractional transformations of simple continued fractions.

3. Word play. Using matrix products to derive results on continued fractions is a natural idea and dates back to at least Kolden $[\mathrm{K}]$. The recurrence formulae can be interpreted as matrix products in at least two (equivalent) ways; we will follow the notation used by Raney in [R].

Let

$$
L=\left(\begin{array}{ll}
1 & 0 \\
1 & 1
\end{array}\right), \quad R=\left(\begin{array}{ll}
1 & 1 \\
0 & 1
\end{array}\right) \quad \text { and } \quad M_{a}=\left(\begin{array}{ll}
a & 0 \\
0 & 1
\end{array}\right)
$$

for any $a \in \mathbb{Z}_{\geq 1}$. The powers of the matrices $L$ and $R$ are simply

$$
L^{z}=\left(\begin{array}{cc}
1 & 0 \\
z & 1
\end{array}\right) \text { and } R^{z}=\left(\begin{array}{ll}
1 & z \\
0 & 1
\end{array}\right)
$$

a notation which we will use for any $z \in \mathbb{R}$ (consistent with defining the non-integral matrix powers using the binomial series). The transpose $(\cdot)^{T}$ and the false transpose $(\cdot)^{\prime}$ of a matrix are defined in the usual way as

$$
\left(\begin{array}{ll}
w & x \\
y & z
\end{array}\right)^{T}=\left(\begin{array}{ll}
w & y \\
x & z
\end{array}\right) \text { and }\left(\begin{array}{ll}
w & x \\
y & z
\end{array}\right)^{\prime}=\left(\begin{array}{ll}
z & x \\
y & w
\end{array}\right) .
$$

Taking both the transpose and the false transpose (in any order) will be referred to as taking the double transpose. Note that $L^{T}=R$.

Given a continued fraction expansion

$$
b_{0}+\frac{a_{1}}{b_{1}}+\frac{a_{2}}{b_{2}}+\cdots
$$

we adopt the shorthand notation

$$
\left\{\begin{array}{l}
E_{2 n}=R^{b_{0}} M_{a_{1}} L^{b_{1}} M_{a_{2}}^{\prime} R^{b_{2}} M_{a_{3}} L^{b_{3}} \cdots M_{a_{2 n}}^{\prime} R^{b_{2 n}}, \\
E_{2 n+1}=R^{b_{0}} M_{a_{1}} L^{b_{1}} M_{a_{2}}^{\prime} R^{b_{2}} M_{a_{3}} L^{b_{3}} \cdots M_{a_{2 n+1}} L^{b_{2 n+1}} .
\end{array}\right.
$$

As with the convergents, when $a_{n}=1$ and $b_{n}=d_{n}$ for all $n$, we will write $E_{n}=F_{n}$ to indicate that the expansion is simple. 
The recurrence formulae of the convergents can be imitated with matrix products:

Lemma 3.1. With the convention $A_{-1}=1, B_{-1}=0$, we have

$$
E_{2 n}=\left(\begin{array}{ll}
A_{2 n-1} & A_{2 n} \\
B_{2 n-1} & B_{2 n}
\end{array}\right), \quad E_{2 n+1}=\left(\begin{array}{ll}
A_{2 n+1} & A_{2 n} \\
B_{2 n+1} & B_{2 n}
\end{array}\right) .
$$

Proof. First,

$$
E_{0}=R^{b_{0}}=\left(\begin{array}{cc}
1 & b_{0} \\
0 & 1
\end{array}\right)=\left(\begin{array}{cc}
A_{-1} & A_{0} \\
B_{-1} & B_{0}
\end{array}\right)
$$

Now if

$$
E_{2 k}=\left(\begin{array}{ll}
A_{2 k-1} & A_{2 k} \\
B_{2 k-1} & B_{2 k}
\end{array}\right)
$$

then

$$
\begin{aligned}
E_{2 k+1} & =E_{2 k} M_{a_{2 k+1}} L^{b_{2 k+1}}=\left(\begin{array}{ll}
A_{2 k-1} & A_{2 k} \\
B_{2 k-1} & B_{2 k}
\end{array}\right)\left(\begin{array}{ll}
a_{2 k+1} & 0 \\
b_{2 k+1} & 1
\end{array}\right) \\
& =\left(\begin{array}{ll}
a_{2 k+1} A_{2 k-1}+b_{2 k+1} A_{2 k} & A_{2 k} \\
a_{2 k+1} B_{2 k-1}+b_{2 k+1} B_{2 k} & B_{2 k}
\end{array}\right)=\left(\begin{array}{ll}
A_{2 k+1} & A_{2 k} \\
B_{2 k+1} & B_{2 k}
\end{array}\right),
\end{aligned}
$$

and if

$$
E_{2 k-1}=\left(\begin{array}{ll}
A_{2 k-1} & A_{2 k-2} \\
B_{2 k-1} & B_{2 k-2}
\end{array}\right)
$$

then

$$
\begin{aligned}
E_{2 k} & =E_{2 k-1} M_{a_{2 k}}^{\prime} R^{b_{2 k}}=\left(\begin{array}{ll}
A_{2 k-1} & A_{2 k-2} \\
B_{2 k-1} & B_{2 k-2}
\end{array}\right)\left(\begin{array}{ll}
1 & b_{2 k} \\
0 & a_{2 k}
\end{array}\right) \\
& =\left(\begin{array}{ll}
A_{2 k-1} & a_{2 k} A_{2 k-2}+b_{2 k} A_{2 k-1} \\
B_{2 k-1} & a_{2 k} B_{2 k-2}+b_{2 k} B_{2 k-1}
\end{array}\right)=\left(\begin{array}{ll}
A_{2 k-1} & A_{2 k} \\
B_{2 k-1} & B_{2 k}
\end{array}\right),
\end{aligned}
$$

and so the claim follows by induction.

By $\operatorname{Sp}(x)=\{t x: t \in \mathbb{R}\}$ we mean the span of a vector in a real vector space. Now suitable matrix products might help us transform a generalized continued fraction expansion into a simple one:

Lemma 3.2. Consider the continued fraction expansions

$$
\tau=b_{0}+\frac{a_{1}}{b_{1}}+\frac{a_{2}}{b_{2}}+\cdots \quad \text { and } \quad \sigma=d_{0}+\frac{1}{d_{1}}+\frac{1}{d_{2}}+\cdots .
$$

If $E_{2 m} \in \operatorname{Sp}\left(F_{2 n}\right)$ or $E_{2 m+1} \in \operatorname{Sp}\left(F_{2 n+1}\right)$ for infinitely many values of $m$ and $n$, then $\tau=\sigma$. 
Proof. By Lemma 3.1 the continued fractions $\tau$ and $\sigma$ have infinitely many common convergents:

or

$$
\frac{A_{2 m-1}}{B_{2 m-1}}=\frac{C_{2 n-1}}{D_{2 n-1}} \quad \text { and } \quad \frac{A_{2 m}}{B_{2 m}}=\frac{C_{2 n}}{D_{2 n}}
$$

$$
\frac{A_{2 m}}{B_{2 m}}=\frac{C_{2 n}}{D_{2 n}} \quad \text { and } \quad \frac{A_{2 m+1}}{B_{2 m+1}}=\frac{C_{2 n+1}}{D_{2 n+1}} .
$$

Then the values of the continued fractions coincide too, as they are the limits of the two convergent sequences.

The matrix products $E_{m}$ and $F_{n}$ can be interpreted as words over the alphabets $\left\{L^{b}, R^{b}, M_{a}, M_{a}^{\prime}: a \in \mathcal{A}, b \in \mathcal{B}\right\}$ and $\{L, R\}$ respectively. Lemmas 3.1 and 3.2 motivate the definition of the legal word set

$$
\operatorname{Lw}(\mathcal{A}, \mathcal{B})=\left\{E_{m}: m \in \mathbb{Z}_{\geq 0}, \tau \in E_{\mathcal{B}}^{\mathcal{A}}\right\} .
$$

On the other hand, define the Kleene star of all the finite products of $L$ and $R$ by

$$
\{L, R\}^{*}=\bigcup_{n \geq 0}\{L, R\}^{n} .
$$

Suppose we have infinitely many $E_{m} \in \operatorname{Sp}\left(F_{n}\right)$, where all $E_{m} \in \operatorname{Lw}(\mathcal{A}, \mathcal{B})$ are prefixes of the same infinite word $W \in\left\{L^{b}, R^{b}, M_{a}, M_{a}^{\prime}: a \in \mathcal{A}, b \in \mathcal{B}\right\}^{\infty}$, and all $F_{n} \in\{L, R\}^{*}$ are prefixes of the same infinite word $V \in\{L, R\}^{\infty}$, cut where the last letter of the prefix and the first letter of the tail are different. The word $W$ can be interpreted as a generalized continued fraction expansion in $E_{\mathcal{B}}^{\mathcal{A}}$, the word $V$ can be interpreted as a simple continued fraction, and by Lemma 3.2 they represent the same number. In fact, for this idea to work, it is not even necessary to require that infinitely many generalized continued fraction convergents coincide with simple convergents, we just do that for simplicity. For instance, Raney did not have such restriction in his algorithm:

Theorem 3.3 (Raney $[\mathrm{R}]$ ). Let $\Delta \in \mathbb{Z}_{\geq 2}$ and define the set of doubly balanced matrices with determinant $\Delta$ as

$\mathcal{D} \mathcal{B}_{\Delta}=\left\{\left(\begin{array}{ll}w & x \\ y & z\end{array}\right): w, x, y, z \in \mathbb{Z}_{\geq 0}, w z-x y=\Delta, \min \{w, z\}>\max \{x, y\}\right\}$.

If $M \in \mathcal{D B}_{\Delta}$ and $W \in\{L, R\}^{\Delta}$, then there exists a non-empty prefix $P$ of $W(P=W$ allowed $), N \in \mathcal{D B}_{\Delta}$ and $Q \in\{L, R\}^{\delta}, 1 \leq \delta \leq \Delta$, such that

$$
M P=Q N \text {. }
$$

The set $\mathcal{D B}_{\Delta}$ is finite, so Raney could use these matrix products to construct a transducer (a finite state automaton that produces an output word) that transforms the infinite word $M W, M \in \mathcal{D} \mathcal{B}_{\Delta}, W \in\{L, R\}^{\infty}$, into 
another infinite word $V \in\{L, R\}^{\infty}$. This is determining the simple continued fraction expansion of the image of another simple continued fraction under the linear fractional transformation corresponding to the matrix $M$ (with some additional theory a bit more flexible than Lemma 3.2.

ExAmple 3.4. Take $\Delta=2$ and $\mathcal{D B}_{2}=\left\{M_{2}, M_{2}^{\prime}\right\}$. Then the matrix products of Theorem 3.3 up to taking the double transpose are

$$
\left\{\begin{array}{l}
M_{2} R=R^{2} M_{2}, \\
M_{2} L^{2}=L M_{2}, \\
M_{2} L R=R L M_{2}^{\prime} .
\end{array}\right.
$$

We may now rewrite the proof of the example given in [HLMT] of the generalized continued fraction Cantor set $E_{\{1\}}^{\{1,2\}}$ containing elements of any prescribed asymptotic irrationality exponent from $\{1\} \cup[2, \infty]$. The proof is the same but this time we keep the underlying word play visible.

Since

$$
M_{2} L M_{2}^{\prime} R L=M_{2} L^{2} R M_{2}=L M_{2} R M_{2}=L R^{2} M_{2}^{2},
$$

for any $h$ we have

$$
\left(M_{2} L M_{2}^{\prime} R\right)^{h} L=L\left(R^{2} M_{2}^{2}\right)^{h}=L R^{f(h)} M_{2}^{2 h},
$$

where

$$
f(h)=2 \cdot 1+2 \cdot 4+\cdots+2 \cdot 4^{h-1}=\frac{2\left(4^{h}-1\right)}{3} .
$$

Taking the false transpose both sides of (3.1) gives

$$
L\left(R M_{2} L M_{2}^{\prime}\right)^{h}=\left(M_{2}^{\prime}\right)^{2 h} R^{f(h)} L,
$$

and therefore

$$
\begin{aligned}
\left(M_{2} L M_{2}^{\prime} R\right)^{h} L R L\left(R M_{2} L M_{2}^{\prime}\right)^{h} R & =L R^{f(h)} M_{2}^{2 h} R\left(M_{2}^{\prime}\right)^{2 h} R^{f(h)} L R \\
& \in \operatorname{Sp}\left(L R^{2 f(h)+4^{h}} L R\right) \cap \operatorname{Lw}(\{1,2\},\{1\}) .
\end{aligned}
$$

By combining blocks like this and using Lemma 3.2 we see that

$$
\frac{1}{1}+\frac{1}{2 f\left(h_{1}\right)+4^{h_{1}}}+\frac{1}{1}+\frac{1}{1}+\frac{1}{1}+\frac{1}{2 f\left(h_{2}\right)+4^{h_{2}}}+\frac{1}{1}+\frac{1}{1}+\cdots \in E_{\{1\}}^{\{1,2\}}
$$

for any sequence $\left(h_{i}\right)_{i=1}^{\infty}$ of positive integers. Obviously we can now make the numbers $d_{n+1}, n \equiv 1(\bmod 4)$, as big as we please regardless of the size of $D_{n}$, so by Lemma 2.1 all the irrationality exponents in $[2, \infty]$ are possible. Finally, the irrationality exponent 1 is also possible since

$$
\frac{2}{1}+\frac{2}{1}+\cdots=1 .
$$

This method is generalized in Theorem 4.2 . 
4. Results. We start by presenting general conditions under which the set $E_{\mathcal{B}}^{\mathcal{A}}$ contains elements with any prescribed asymptotic irrationality exponent. In Example 3.4 the numbers constructed were quite close to the expansion

$$
1=\frac{2}{1}+\frac{2}{1}+\cdots
$$

This raises the question whether it would be possible to construct our numbers from rational numbers in $E_{\mathcal{B}}^{\mathcal{A}}$. It turns out that under a few assumptions it is:

Lemma 4.1. Let $p / q \in E_{\mathcal{B}}^{\mathcal{A}}$ be a rational number with a periodic generalized and a finite simple expansion

$$
\frac{p}{q}=\frac{a_{1}}{b_{1}}+\frac{a_{2}}{b_{2}}+\cdots+\frac{a_{m}}{b_{m}}+\frac{a_{1}}{b_{1}}+\frac{a_{2}}{b_{2}}+\cdots=d_{0}+\frac{1}{d_{1}}+\frac{1}{d_{2}}+\cdots+\frac{1}{d_{n}},
$$

where $m$ is the least possible even period length. Denote $\lambda_{1}=B_{m}+p B_{m-1} / q$, $\lambda_{2}=B_{m}-q A_{m} / p, \Lambda=\operatorname{diag}\left(\lambda_{1}, \lambda_{2}\right)$ and

$$
k=\frac{q A_{m} D_{n-1}+p B_{m-1} C_{n-1}}{p q B_{m}-q^{2} A_{m}} .
$$

Then

$$
E_{m} F_{n}= \begin{cases}F_{n} R^{k} \Lambda & \text { when } n \text { is odd, } \\ F_{n} L^{k} \Lambda^{\prime} & \text { when } n \text { is even } .\end{cases}
$$

Proof. Assume that $n$ is odd. By the periodicity of the generalized continued fraction expansion of $p / q$ we have

$$
\frac{p}{q}=\frac{p A_{m-1}+q A_{m}}{p B_{m-1}+q B_{m}} .
$$

It can now be easily verified that $\lambda_{1}$ and $\lambda_{2}$ are the eigenvalues of the matrix $E_{m}$ with corresponding eigenvectors $(p, q)$ and $\left(q A_{m},-p B_{m-1}\right)$, respectively. Since $\lambda_{1} \neq \lambda_{2}$, the eigenvectors are linearly independent and

$$
E_{m} Q=Q \Lambda
$$

where

$$
Q=\left(\begin{array}{cc}
p & q A_{m} \\
q & -p B_{m-1}
\end{array}\right) .
$$

Since $C_{n}=p$ and $D_{n}=q$, by (2.1) we have

$$
Q=F_{n} W=F_{n}\left(\begin{array}{cc}
1 & x \\
0 & -y
\end{array}\right),
$$


where $x=q A_{m} D_{n-1}+p B_{m-1} C_{n-1}$ and $y=q^{2} A_{m}+p^{2} B_{m-1}$. Further

$$
\begin{aligned}
W \Lambda W^{-1} & =\left(\begin{array}{cc}
1 & x \\
0 & -y
\end{array}\right)\left(\begin{array}{cc}
\lambda_{1} & 0 \\
0 & \lambda_{2}
\end{array}\right)\left(\begin{array}{cc}
1 & x / y \\
0 & -1 / y
\end{array}\right)=\left(\begin{array}{cc}
\lambda_{1} & x\left(\lambda_{1}-\lambda_{2}\right) / y \\
0 & \lambda_{2}
\end{array}\right) \\
& =\left(\begin{array}{cc}
1 & x\left(\lambda_{1}-\lambda_{2}\right) /\left(\lambda_{2} y\right) \\
0 & 1
\end{array}\right)\left(\begin{array}{cc}
\lambda_{1} & 0 \\
0 & \lambda_{2}
\end{array}\right)=R^{k} \Lambda .
\end{aligned}
$$

It follows from equations $4.1-4.3$ that

$$
E_{m} F_{n}=F_{n} W \Lambda W^{-1}=F_{n} R^{k} \Lambda .
$$

The proof for the case when $n$ is even is analogous.

TheOREM 4.2. Let $p / q \in E_{\mathcal{B}}^{\mathcal{A}}$ be a rational number with a periodic generalized and a finite simple expansion

$$
\frac{p}{q}=\frac{a_{1}}{b_{1}}+\frac{a_{2}}{b_{2}}+\cdots+\frac{a_{m}}{b_{m}}+\frac{a_{1}}{b_{1}}+\frac{a_{2}}{b_{2}}+\cdots=d_{0}+\frac{1}{d_{1}}+\frac{1}{d_{2}}+\cdots+\frac{1}{d_{n}},
$$

where $m$ is the least possible even period length. Denote $\lambda_{1}=B_{m}+p B_{m-1} / q$, $\lambda_{2}=B_{m}-q A_{m} / p$ and

$$
k=\frac{q A_{m} D_{n-1}+p B_{m-1} C_{n-1}}{p q B_{m}-q^{2} A_{m}},
$$

and require that $\lambda=\lambda_{1} / \lambda_{2}$ and $2 k$ are integers. Further, denote $Y=R$ when $n$ is odd and $Y=L$ when $n$ is even.

If there exist $l \in \mathbb{Q}_{\geq 0}$ and $T \in \operatorname{Sp}\left(F_{n} Y^{l} F_{n}^{\prime}\right)$ such that $\lambda^{h} l \in \mathbb{Z}$ for all $h \in \mathbb{Z}_{\geq 1}$ big enough and $E_{m} T E_{m}^{\prime} R^{b} \in \operatorname{Lw}(\mathcal{A}, \mathcal{B})$ for some $b \in \mathcal{B}$, then for any $s \in\{1\} \cup[2, \infty]$ there exists $\tau_{s} \in E_{\mathcal{B}}^{\mathcal{A}}$ with asymptotic irrationality exponent $\mu_{I}\left(\tau_{s}\right)=s$.

Proof. Since $p / q \in E_{\mathcal{B}}^{\mathcal{A}}$, there is an element in $E_{\mathcal{B}}^{\mathcal{A}}$ with asymptotic irrationality exponent $s=1$.

Assume that $n$ is odd.

Denote $\Lambda=\operatorname{diag}\left(\lambda_{1}, \lambda_{2}\right)$. By Lemma 4.1 we have $E_{m} F_{n}=F_{n} R^{k} \Lambda$. Since $\Lambda R^{z}=R^{z \lambda} \Lambda$, for all $h \in \mathbb{Z}_{\geq 1}$ we inductively get

$$
E_{m}^{h} F_{n}=F_{n} R^{k\left(1+\lambda+\cdots+\lambda^{h-1}\right)} \Lambda^{h} .
$$

Using false transpose gives

$$
E_{m}^{h} F_{n} R^{l} F_{n}^{\prime}\left(E_{m}^{\prime}\right)^{h} \in \operatorname{Sp}\left(F_{n} R^{f(h)} F_{n}^{\prime}\right),
$$

where $f(h)=2 k\left(1+\lambda+\cdots+\lambda^{h-1}\right)+\lambda^{h} l$. Since $A_{m} / B_{m}<p / q$ for even $m$, we have $\lambda_{2}>0$ and $k>0$. Hence our assumptions imply that $f(h) \in \mathbb{Z}_{\geq 1}$ for all large enough $h \in \mathbb{Z}_{\geq 1}$. We get

$$
\begin{aligned}
E_{m}^{h} T\left(E_{m}^{\prime}\right)^{h} R^{b} & \in \operatorname{Sp}\left(E_{m}^{h} F_{n} R^{l} F_{n}^{\prime}\left(E_{m}^{\prime}\right)^{h} R^{b}\right) \cap \operatorname{Lw}(\mathcal{A}, \mathcal{B}) \\
& =\operatorname{Sp}\left(F_{n} R^{f(h)} F_{n}^{\prime} R^{b}\right) \cap \operatorname{Lw}(\mathcal{A}, \mathcal{B})
\end{aligned}
$$


for any $b \in \mathcal{B}$. Using (4.4) and Lemma 3.2 we can now construct generalized continued fractions in $E_{\mathcal{B}}^{\mathcal{A}}$ with known simple continued fraction expansions.

Let $s \in \mathbb{R}_{\geq 2}$ and denote $N=2 n+2$. Consider the simple continued fraction

$$
\tau_{s}=\hat{d}_{0}+\frac{1}{\hat{d}_{1}}+\frac{1}{\hat{d}_{2}}+\cdots
$$

with

$$
\hat{d}_{i}= \begin{cases}d_{j} & \text { when } i \equiv j(\bmod N) \text { and } j \in\{1, \ldots, n\} \\ f\left(g_{i}\right) & \text { when } i \equiv n+1(\bmod N), \\ d_{N-j} & \text { when } i \equiv j(\bmod N) \text { and } j \in\{n+2, n+3, \ldots, 2 n+1\} \\ 2 d_{0}+b & \text { when } i \equiv 0(\bmod N)\end{cases}
$$

and

$$
g_{i}=\left\lceil\frac{\log \frac{\hat{D}_{i-1}^{s-2}+\beta}{l+\beta}}{\log \lambda}\right\rceil, \quad \beta=\frac{2 k}{\lambda-1},
$$

where $\hat{D}_{i}$ is the denominator of the $i$ th convergent of $\tau_{s}$. By Lemma 2.1 the asymptotic irrationality exponent of a simple continued fraction is determined by its tail, and so we may assume that $\hat{d}_{i} \in \mathbb{Z}$ for all $i$, omitting some multiple of $N$ first $\hat{d}_{i}$ if the first values of $f\left(g_{i}\right)$ are not integral. By equation 4.4 the number $\tau_{s}$ is in $E_{\mathcal{B}}^{\mathcal{A}}$. Since the partial denominators $\hat{d}_{i}$ are bounded when $i \not \equiv n+1(\bmod N)$ and

$$
\hat{D}_{i-1}^{s-2} \leq \hat{d}_{i} \leq \lambda \hat{D}_{i-1}^{s-2}+2 k
$$

when $i \equiv n+1(\bmod N)$, we get

$$
\limsup _{i \rightarrow \infty} \frac{\log \hat{d}_{i+1}}{\log \hat{D}_{i}}=\limsup _{j \rightarrow \infty} \frac{\log \hat{d}_{N j+n+1}}{\log \hat{D}_{N j+n}}=s-2 .
$$

Hence $\mu\left(\tau_{s}\right)=s$ by Lemma 2.1 .

When we define $\tau_{\infty}$ by replacing $s$ with $i$ in 4.5 , we have

$$
\hat{D}_{i-1}^{i-2} \leq \hat{d}_{i} \leq \lambda \hat{D}_{i-1}^{i-2}+2 k
$$

when $i \equiv n+1(\bmod N)$, so

$$
\limsup _{i \rightarrow \infty} \frac{\log \hat{d}_{i+1}}{\log \hat{D}_{i}}=\limsup _{j \rightarrow \infty} \frac{\log \hat{d}_{N j+n+1}}{\log \hat{D}_{N j+n}}=\infty,
$$

and hence $\tau_{\infty}$ is a Liouville number.

The proof for $n$ even is analogous.

Example 4.3. Consider

$$
\frac{4}{7}=\frac{6}{7}+\frac{30}{8}+\overline{7}+\overline{8} \frac{30}{8}+\cdots=\frac{1}{1}+\frac{1}{1}+\frac{1}{3} \in E_{\{6,7,8\}}^{\{6,30\}} .
$$


Now we have $\lambda_{1}=90, \lambda_{2}=2$ and $k=25 / 2$. The choices $l=14 / 5$ and

$$
T=M_{6} L^{8} M_{30}^{\prime} R^{7} M_{30} L^{6} M_{6}^{\prime} R^{8} M_{6} L^{6} M_{30}^{\prime} R^{7} M_{30} L^{8} M_{6}^{\prime}=180^{2} F_{3} R^{14 / 5} F_{3}^{\prime}
$$

fulfill the conditions of Theorem 4.2 . Hence the set $E_{\{6,7,8\}}^{\{6,30\}}$ contains numbers for any possible asymptotic irrationality exponent.

Despite acquiring all the possible asymptotic irrationality exponents, a generalized continued fraction Cantor set can be arbitrarily small in the sense of Hausdorff dimension. Recall that if $F \subseteq \mathbb{R}$ and

$$
H_{\delta}^{s}(F)=\inf \left\{\sum_{i=1}^{\infty} \operatorname{diam}\left(A_{i}\right)^{s}: F \subseteq \bigcup_{i=1}^{\infty} A_{i}, \operatorname{diam}\left(A_{i}\right) \leq \delta \text { for all } i\right\},
$$

where $\operatorname{diam}\left(A_{i}\right)=\sup \left\{|x-y|: x, y \in A_{i}\right\}$, and $H^{s}(F)=\lim _{\delta \rightarrow 0} H_{\delta}^{s}(F)$, then the Hausdorff dimension of $F$ is defined as

$$
\operatorname{dim}_{H}(F)=\inf \left\{t: H^{t}(F)<\infty\right\} .
$$

Theorem 4.4. Let $a \in \mathbb{Z}_{\geq 3}, \mathcal{A}=\{a\}$ and $\mathcal{B}=\{a-1, b\}$, where $b=a$ or $b=2 a$. Then for any $s \in\{1\} \cup[2, \infty]$ there exists $\tau_{s} \in E_{\mathcal{B}}^{\mathcal{A}}$ with asymptotic irrationality exponent $\mu_{I}\left(\tau_{s}\right)=s$. Further, $\lim _{a \rightarrow \infty} \operatorname{dim}_{H}\left(E_{\mathcal{B}}^{\mathcal{A}}\right)=0$.

Proof. The Cantor set $E_{\mathcal{B}}^{\mathcal{A}}$ contains the rational number

$$
\frac{a}{a-1}+\frac{a}{a-1}+\cdots=1 \in E_{\mathcal{B}}^{\mathcal{A}},
$$

which gives

$$
E_{2}=\left(\begin{array}{cc}
a & a(a-1) \\
a-1 & a^{2}-a+1
\end{array}\right), \quad F_{0}=\left(\begin{array}{ll}
1 & 1 \\
0 & 1
\end{array}\right)
$$

and we calculate $\lambda_{1}=a^{2}, \lambda_{2}=1$ and $k=a-1$. For $b=a$ the choices $l=1$ and $T=R M_{a} L^{a} M_{a}^{\prime} R=a F_{0} L F_{0}^{\prime}$ fulfill the conditions of Theorem 4.2. For $b=2 a$ we choose $l=0$ and $T=R^{2}$.

Let $T_{1}(x)=a /\left(b_{1}+x\right)=a /(b+x), T_{2}(x)=a /\left(b_{2}+x\right)=a /(a-1+x)$ and $I=\left[\tau_{m}, \tau_{M}\right]$, where

$$
\begin{aligned}
\tau_{m} & =\min E_{a}=\frac{a}{b}+\frac{a}{a-1}+\frac{a}{b}+\frac{a}{a-1}+\cdots \\
& =\frac{-(a-1)+(a-1) \sqrt{1+\frac{4 a}{b(a-1)}}}{2}, \\
\tau_{M} & =\max E_{a}=\frac{a}{a-1+\tau_{m}}=\frac{-b+b \sqrt{1+\frac{4 a}{b(a-1)}}}{2} .
\end{aligned}
$$

For $\underline{i}=\left(i_{1}, \ldots, i_{K}\right), i_{j} \in\{1,2\}$, denote $T_{\underline{\underline{i}}}(x)=\left(T_{i_{1}} \circ \cdots \circ T_{i_{K}}\right)(x)$. Now

$$
E_{\mathcal{B}}^{\mathcal{A}}=\bigcap_{K \geq 1} \bigcup_{|\underline{i}|=K} T_{\underline{i}}(I) \Rightarrow E_{\mathcal{B}}^{\mathcal{A}} \subseteq \bigcup_{|\underline{i}|=K} T_{\underline{i}}(I)
$$


for all $K \geq 1$. Also $T_{\underline{i}}(I) \subset I$ for all $\underline{i}$. Since

$$
\begin{aligned}
\left|T_{i}(x)-T_{i}(y)\right| & =\left|\frac{a}{b_{i}+x}-\frac{a}{b_{i}+y}\right|=\frac{a|x-y|}{\left(b_{i}+x\right)\left(b_{i}+y\right)} \\
& \leq \frac{a}{(a-1)^{2}} \underbrace{\left|\tau_{M}-\tau_{m}\right|}_{<1}<\frac{a}{(a-1)^{2}}<1
\end{aligned}
$$

for $i \in\{1,2\}$ and $x, y \in I$, inductively $\left|T_{\underline{\underline{i}}}\left(\tau_{m}\right)-T_{\underline{i}}\left(\tau_{M}\right)\right|<\left(a /(a-1)^{2}\right)^{K}$ for every $\underline{i}$ such that $|\underline{i}|=K \geq 1$.

Now if $\delta>0$, choose $K \geq 1$ such that $\left(a /(a-1)^{2}\right)^{K} \leq \delta$. Then

$$
\begin{aligned}
H_{\delta}^{s}\left(E_{\mathcal{B}}^{\mathcal{A}}\right) & \leq \sum_{|\underline{i}|=K} \operatorname{diam}\left(T_{\underline{i}}(I)\right)^{s}<\sum_{|\underline{i}|=K}\left(\frac{a}{(a-1)^{2}}\right)^{K s} \\
& =2^{K}\left(\frac{a}{(a-1)^{2}}\right)^{K s}=\left(2\left(\frac{a}{(a-1)^{2}}\right)^{s}\right)^{K} .
\end{aligned}
$$

When

$$
2\left(\frac{a}{(a-1)^{2}}\right)^{s}=1 \Leftrightarrow s=\frac{\log 2}{\log \left((a-1)^{2} / a\right)},
$$

then $H_{\delta}^{s}\left(E_{\mathcal{B}}^{\mathcal{A}}\right) \leq 1^{K}=1$ for all $\delta>0$, so $H^{s}\left(E_{\mathcal{B}}^{\mathcal{A}}\right)=\lim _{\delta \rightarrow 0} H_{\delta}^{s}\left(E_{\mathcal{B}}^{\mathcal{A}}\right) \leq 1$. Hence

$$
\operatorname{dim}_{H}\left(E_{\mathcal{B}}^{\mathcal{A}}\right) \leq \frac{\log 2}{\log \left((a-1)^{2} / a\right)}
$$

and $\operatorname{dim}_{H}\left(E_{\mathcal{B}}^{\mathcal{A}}\right) \rightarrow 0$ as $a \rightarrow \infty$.

In the assumptions of Theorem 4.2 the matrix $T$ can be replaced by $\hat{T}$ if $E_{m} \hat{T} E_{m}^{\prime} R^{b} \in \operatorname{Lw}(\mathcal{A}, \mathcal{B})$ and $\hat{T} \in \operatorname{Sp}\left(F_{n} \hat{H} F_{n}^{\prime}\right)$, where

$$
R^{k\left(1+\lambda+\cdots+\lambda^{h-1}\right)} \Lambda^{h} \hat{H}\left(\Lambda^{\prime}\right)^{h} R^{k\left(1+\lambda+\cdots+\lambda^{h-1}\right)} \in \operatorname{Sp}\left(V_{h}\right)
$$

and $V_{h} \in\{L, R\}^{*}$ contains as a subword an increasing power of $L$ or $R$ with respect to $h$, otherwise being of bounded length. The length of $V_{h}$ must be controlled so that in the construction we can choose $\hat{d}_{i+1}$ to be arbitrarily large regardless of $\hat{D}_{i}$. Still, as long as the growth rate of the length of the word $V_{h}$ without the increasing power of $L$ or $R$ is linear and the growth rate of the exponent in the increasing power is exponential, the resulting approximants remain unusually while not arbitrarily good. This is the basic idea of our last theorem:

TheOrem 4.5. Let $\mathcal{A}, \mathcal{B}$ be finite sets of positive integers and $\Delta \in \mathbb{Z}_{\geq 2}$ be such that $b \Delta^{h} / a \in \mathbb{Z}$ for some $a \in \mathcal{A}, b \in \mathcal{B}$ and $h \in \mathbb{Z}_{\geq 1}$ large enough. Define

$\mathcal{D B}_{\Delta}=\left\{\left(\begin{array}{ll}w & x \\ y & z\end{array}\right): w, x, y, z \in \mathbb{Z}_{\geq 0}, w z-x y=\Delta, \min \{w, z\}>\max \{x, y\}\right\}$. 
Suppose that for any $X \in \mathcal{D B}_{\Delta}, P \in \bigcup_{\delta<\Delta}\{L, R\}^{\delta}$, and $Y \in\{L, R\}$ there exist $W_{Y}(X, P)$ such that either $W_{Y}(X, P)$ or its double transpose is in $\operatorname{Lw}(\mathcal{A}, \mathcal{B})$ and $W_{Y}(X, P)$ ends with $Y$, and $U_{Y}(X, P) \in\{L, R\}^{*}$ such that

$$
X P W_{Y}(X, P)^{\prime}=\sqrt{\operatorname{det}\left(X P W_{Y}(X, P)^{\prime}\right)} U_{Y}(X, P)^{\prime} .
$$

Define

$$
\Gamma=\max _{X, P, Y}\left\{\frac{1}{\sqrt{\operatorname{det}\left(X P W_{Y}(X, P)^{\prime}\right)}}\left\|W_{Y}(X, P)\right\|_{\infty}\right\},
$$

where the $L_{\infty}$-norm $\|\cdot\|_{\infty}$ means the maximal row sum. Then there exists $\tau \in E_{\mathcal{B}}^{\mathcal{A}}$ with

$$
\mu_{I}(\tau) \geq 2+\frac{\log \Delta}{\log (\Delta \Gamma)}>2 .
$$

Proof. For convenience, denote

$$
\overline{\mathrm{Lw}}(\mathcal{A}, \mathcal{B})=\operatorname{Lw}(\mathcal{A}, \mathcal{B}) \cup\left\{E_{m}^{\prime T}: m \in \mathbb{Z}_{\geq 0}, \tau \in E_{\mathcal{B}}^{\mathcal{A}}\right\} .
$$

We start by constructing infinite sequences $\left(W_{i}\right)_{i=1}^{\infty}$ consisting of the matrices $W_{Y}(X, P)$, and $\left(V_{i}\right)_{i=1}^{\infty}$ with $V_{i} \in\{L, R\}^{*}$, in such a way that

$$
W_{h} W_{h-1} \cdots W_{1} \in \operatorname{Sp}\left(V_{h} M_{\Delta}^{h}\right) \cap \overline{\mathrm{Lw}}(\mathcal{A}, \mathcal{B})
$$

for all $h \in \mathbb{Z}_{\geq 1}$. Set

$$
\left\{\begin{array}{l}
W_{1}=W_{L}\left(M_{\Delta}, I\right) \\
V_{1}=U_{L}\left(M_{\Delta}, I\right)
\end{array}\right.
$$

Then $W_{1} \in \overline{\mathrm{Lw}}(\mathcal{A}, \mathcal{B})$, and taking the false transpose of (4.6) with $X=M_{\Delta}$ and $P=I$ and multiplying by $M_{\Delta}$ from the right yields $W_{1} \in \operatorname{Sp}\left(V_{1} M_{\Delta}\right)$.

Suppose the matrices $W_{k}$ and $V_{k}$ are defined and (4.7) is true for $k \leq h$. By Raney's Theorem 3.3 there exists $X \in \mathcal{D B}_{\Delta}$ and $P \in \bigcup_{\delta<\Delta}\{L, R\}^{\delta}$ such that

$$
M_{\Delta} V_{h}^{\prime}=T_{h}^{\prime} X P, \quad T_{h} \in\{L, R\}^{*} .
$$

Choose $Y \in\{L, R\}$ in such a way that $W_{Y}(X, P) W_{h} \in \overline{\mathrm{Lw}}(\mathcal{A}, \mathcal{B})$ and define

$$
\left\{\begin{array}{l}
W_{h+1}=W_{Y}(X, P) \\
V_{h+1}=U_{Y}(X, P) T_{h}
\end{array}\right.
$$

Now $W_{h+1} W_{h} \cdots W_{1} \in \overline{\mathrm{Lw}}(\mathcal{A}, \mathcal{B})$ by construction. The condition 4.6$)$ gives

$$
\begin{aligned}
\sqrt{\operatorname{det}\left(X P W_{h+1}^{\prime}\right)} V_{h+1}^{\prime} & =\sqrt{\operatorname{det}\left(X P W_{Y}(X, P)^{\prime}\right)} T_{h}^{\prime} U_{Y}(X, P)^{\prime} \\
& =T_{h}^{\prime} X P W_{Y}(X, P)^{\prime}=M_{\Delta} V_{h}^{\prime} W_{h+1}^{\prime} .
\end{aligned}
$$

Now we can use the induction hypothesis 4.7) to obtain

$$
W_{h+1} W_{h} \cdots W_{1} \in \operatorname{Sp}\left(W_{h+1} V_{h} M_{\Delta}^{h}\right)=\operatorname{Sp}\left(V_{h+1} M_{\Delta}^{h+1}\right) .
$$


We can mirror the left hand side of (4.7) using the false transpose and glue the pieces together with permitted blocks $M_{a}^{\prime} R^{b} M_{a}$ and $Y_{h} \in\left\{L^{b}, R^{b}\right\}$ to get

$$
S_{h}=W_{h} W_{h-1} \cdots W_{1} M_{a}^{\prime} R^{b} M_{a} W_{1}^{\prime} \cdots W_{h-1}^{\prime} W_{h}^{\prime} Y_{h} \in \overline{\mathrm{Lw}}(\mathcal{A}, \mathcal{B}) .
$$

Using (4.7) and the matrix identities $M_{z} R=R^{z} M_{z}$ and $M_{z} M_{z}^{\prime}=z I$ we deduce

$$
S_{h} \in \operatorname{Sp}\left(V_{h} R^{b \Delta^{h} / a} V_{h}^{\prime} Y_{h}\right) .
$$

Consider the generalized continued fraction $\tau$ corresponding to the infinite word $\hat{S}_{g_{1}} \hat{S}_{g_{2}} \ldots$, where the sequence $\left(g_{i}\right)_{i=1}^{\infty}$ will be described shortly and $\hat{S}_{g_{i}}$ stands for either $S_{g_{i}}$ or its double transpose, determined by the requirement $\hat{S}_{g_{1}} \cdots \hat{S}_{g_{i}} \in \operatorname{Lw}(\mathcal{A}, \mathcal{B})$ for all $i$. By the construction we have $\tau \in E_{\mathcal{B}}^{\mathcal{A}}$, and provided that all the numbers $b \Delta^{g_{i}} / a$ are integral, Lemma 3.2 together with 4.9) describes the simple continued fraction expansion

$$
\tau=d_{0}+\frac{1}{d_{1}}+\frac{1}{d_{2}}+\cdots
$$

of $\tau$. Define the sequence $\left(\gamma_{i}+1\right)_{i=1}^{\infty}$ of the indices of the exceptionally high partial numerators in 4.9 so that $d_{\gamma_{i}+1} \geq b \Delta^{g_{i}} / a$. This inequality might be strict because $V_{g_{i}}$ could end with $R$, making the partial numerator $d_{\gamma_{i}+1}$ higher. However, if $V_{g_{i}}$ was a perfect power of $R$, the bottom left element of $V_{g_{i}} M_{\Delta}^{g_{i}}$ would be zero. When $g_{i}>1$, this is impossible by (4.7), because no $W_{Y}(X, P)$ is the identity matrix. This means that the indices $\gamma_{i}+1$ form a well defined strictly increasing sequence. In order to use Lemma 2.1 we need to estimate $D_{\gamma_{i}}$ in terms of $g_{i}$.

We begin by estimating the elements of $V_{h}$. From (4.8) we get

$$
V_{h+1}=\frac{1}{\sqrt{\operatorname{det}\left(X P W_{Y}(X, P)^{\prime}\right)}} W_{Y}(X, P) V_{h} M_{\Delta}^{\prime} .
$$

If we label the elements of $W_{Y}(X, P)$ by $w_{i j}$ and the elements of $V_{h}$ by $v_{i j}$, this becomes

$$
V_{h+1}=\frac{1}{\sqrt{\operatorname{det}\left(X P W_{Y}(X, P)^{\prime}\right)}}\left(\begin{array}{ll}
w_{11} v_{11}+w_{12} v_{21} & \Delta\left(w_{11} v_{12}+w_{12} v_{22}\right) \\
w_{21} v_{11}+w_{22} v_{21} & \Delta\left(w_{21} v_{12}+w_{22} v_{22}\right)
\end{array}\right),
$$

meaning that the maximal element of $V_{h+1}$ is at most $\Delta$ times $\Gamma$ times the maximal element of $V_{h}$. In other words, the elements of $V_{h}$ are bounded from above by $(\Delta \Gamma)^{h}$.

By Lemma 3.1, $D_{\gamma_{i}}$ is an element of the matrix $F_{\gamma_{i}}=Z_{i-1} \hat{V}_{g_{i}}$, where

$$
Z_{i-1}=\frac{1}{\sqrt{\operatorname{det}\left(\hat{S}_{g_{1}} \cdots \hat{S}_{g_{i-1}}\right)}} \hat{S}_{g_{1}} \cdots \hat{S}_{g_{i-1}}
$$


and $\hat{V}_{g_{i}}$ is either $V_{g_{i}}$ or its double transpose. If $z_{i-1}$ stands for the maximal element of $Z_{i-1}$ and $v_{g_{i}}$ for the maximal element of $\hat{V}_{g_{i}}$ (also the maximal element of $\left.V_{g_{i}}\right)$, we get the bound $D_{\gamma_{i}} \leq 2 z_{i-1} v_{g_{i}} \leq 2 z_{i-1}(\Delta \Gamma)^{g_{i}}$. Now

$$
\frac{\log d_{\gamma_{i}+1}}{\log D_{\gamma_{i}}} \geq \frac{\log b \Delta^{g_{i}} / a}{\log \left(2 z_{i-1}(\Delta \Gamma)^{g_{i}}\right)}=\frac{\log b-\log a+g_{i} \log \Delta}{\log 2+\log z_{i-1}+g_{i} \log (\Delta \Gamma)} .
$$

The choice of $g_{i}$ is completely independent of the number $z_{i-1}$, so we can pick any $g_{1}$ satisfying $b \Delta^{g_{1}} / a \in \mathbb{Z}$ and each $g_{i}, i>1$, so large that $b \Delta^{g_{i}} / a \in \mathbb{Z}$ and the difference between the right hand side of (4.10) and $\log \Delta / \log (\Delta \Gamma)$ is at most $1 / i$. The claim follows from Lemma 2.1 after estimating the lim sup by the lower bound of the limit of the subsequence indexed by $\gamma_{i}$.

In particular, there exist generalized continued fraction Cantor sets that contain very well approximable numbers but no Liouville numbers.

ExAmple 4.6. Let $a, b \in \mathbb{Z}_{\geq 1}, \mathcal{A}=\{a, 2 a\}, \mathcal{B}=\{b a\}$ and $\Delta=2$. Then we have $\mathcal{D B}_{2}=\left\{M_{2}, M_{2}^{\prime}\right\}$ and $\bigcup_{\delta<2}\{L, R\}^{\delta}=\{I, L, R\}$. We will need the equations

$$
M_{2} L R=R L M_{2}^{\prime}, \quad M_{z} L^{z}=L M_{z},
$$

and the equations obtained from these by taking the transpose and/or the false transpose of both sides.

If $b a$ is odd, the matrices $W_{Y}\left(M_{2}, P\right)$ and $U_{Y}\left(M_{2}, P\right)$ required in Theorem 4.5 are:

\begin{tabular}{cccc}
\hline$Y$ & $P$ & $W_{Y}\left(M_{2}, P\right)$ & $U_{Y}\left(M_{2}, P\right)$ \\
\hline$L$ & $I$ & $M_{2 a}^{\prime} R^{b a} M_{a} L^{b a}$ & $R^{(b-1) / 2} L R L^{(b a-1) / 2}$ \\
$R$ & $I$ & $M_{a} L^{b a} M_{2 a}^{\prime} R^{b a} M_{a} L^{b a} M_{a}^{\prime} R^{b a}$ & $L^{b} R^{(b a-1) / 2} L R L^{(b-1) / 2} R^{2 b a}$ \\
$L$ & $L$ & $M_{a}^{\prime} R^{b a} M_{2 a} L^{b a}$ & $R^{b} L^{(b a+1) / 2}$ \\
$R$ & $L$ & $M_{a} L^{b a} M_{2 a}^{\prime} R^{b a}$ & $L^{b} R^{(b a-1) / 2} L R$ \\
$L$ & $R$ & $M_{2 a}^{\prime} R^{b a} M_{a} L^{b a}$ & $R^{(b-1) / 2} L R L^{(b a-1) / 2} R^{2}$ \\
$R$ & $R$ & $M_{a} L^{b a} M_{2 a}^{\prime} R^{b a} M_{a} L^{b a} M_{a}^{\prime} R^{b a}$ & $L^{b} R^{(b a-1) / 2} L R L^{(b-1) / 2} R^{2 b a+2}$ \\
\hline
\end{tabular}

Assume next that $b a$ is even. When $b$ is odd, the matrices $W_{Y}\left(M_{2}, P\right)$ and $U_{Y}\left(M_{2}, P\right)$ are:

\begin{tabular}{cccc}
\hline$Y$ & $P$ & $W_{Y}\left(M_{2}, P\right)$ & $U_{Y}\left(M_{2}, P\right)$ \\
\hline$L$ & $I$ & $M_{a}^{\prime} R^{b a} M_{2 a} L^{b a}$ & $R^{b} L^{b a / 2}$ \\
$R$ & $I$ & $M_{2 a} L^{b a} M_{a}^{\prime} R^{b a} M_{a} L^{b a} M_{a}^{\prime} R^{b a}$ & $L^{(b-1) / 2} R L R^{(b a-2) / 2} L R L^{(b-1) / 2} R^{2 b a}$ \\
$L$ & $L$ & $M_{2 a}^{\prime} R^{b a} M_{a} L^{b a}$ & $R^{(b-1) / 2} L R L^{b a / 2}$ \\
$R$ & $L$ & $M_{2 a} L^{b a} M_{a}^{\prime} R^{b a}$ & $L^{(b-1) / 2} R L R^{(b a-2) / 2} L R$ \\
$L$ & $R$ & $M_{a}^{\prime} R^{b a} M_{2 a} L^{b a}$ & $R^{b} L^{b a / 2} R^{2}$ \\
$R$ & $R$ & $M_{2 a} L^{b a} M_{a}^{\prime} R^{b a} M_{a} L^{b a} M_{a}^{\prime} R^{b a}$ & $L^{(b-1) / 2} R L R^{(b a-2) / 2} L R L^{(b-1) / 2} R^{2 b a+2}$ \\
\hline
\end{tabular}


The case of $b$ even is a bit more complicated. Denote

$$
G=M_{2 a}^{\prime} R^{b a} M_{a} L^{b a} .
$$

Let $b=2^{n} c$, where $n \in \mathbb{Z}_{\geq 1}$ and $c$ is odd. It can be shown by induction that

$$
G^{k}=a^{k}\left(\prod_{i=1}^{k} R^{2^{n-i} c} L^{2^{n+i} a c}\right) M_{2^{k}}^{\prime}=a^{k} J_{k} M_{2^{k}}^{\prime}
$$

for all $0 \leq k \leq n$. Then

$$
\begin{aligned}
M_{2} L G^{k} & =a^{k}\left(\prod_{i=1}^{k} R L R^{2^{n-i-1} c-1} L R L^{2^{n+i-1} a c-1}\right) M_{2} L M_{2^{k}}^{\prime} \\
& =a^{k} K_{k} M_{2} L M_{2^{k}}^{\prime} .
\end{aligned}
$$

Now the matrices $W_{Y}\left(M_{2}, P\right)$ and $U_{Y}\left(M_{2}, P\right)$ are:

\begin{tabular}{cccc}
\hline$Y$ & $P$ & $W_{Y}\left(M_{2}, P\right)$ & $U_{Y}\left(M_{2}, P\right)$ \\
\hline$L$ & $I$ & $M_{a}^{\prime} R^{b a} M_{2 a} L^{b a}$ & $R^{b} L^{b a / 2}$ \\
$R$ & $I$ & $M_{2 a} L^{b a} M_{a}^{\prime} R^{b a}$ & $L^{b / 2} R^{2 b a}$ \\
$L$ & $L$ & $G^{n} M_{2 a}^{\prime} R^{b a} M_{2 a}\left(G^{\prime}\right)^{n-1} L^{b a}$ & $J_{n} R^{(c-1) / 2} L R K_{n-1}^{\prime} L^{b a / 2}$ \\
$R$ & $L$ & $\left(G^{\prime T}\right)^{n} M_{2 a} L^{b a} M_{2 a}^{\prime}\left(G^{T}\right)^{n-1} R^{b a}$ & $J_{n}^{\prime T} L^{(c-1) / 2} R L K_{n-1}^{T} R^{(b a-2) / 2} L R$ \\
$L$ & $R$ & $M_{a}^{\prime} R^{b a} M_{2 a} L^{b a}$ & $R^{b} L^{b a / 2} R^{2}$ \\
$R$ & $R$ & $M_{2 a} L^{b a} M_{a}^{\prime} R^{b a}$ & $L^{b / 2} R^{2 b a+2}$ \\
\hline
\end{tabular}

In all the cases above we may choose

$$
\begin{gathered}
W_{Y}\left(M_{2}^{\prime}, P\right)=W_{Y^{T}}\left(M_{2}, P^{\prime T}\right)^{\prime T}, \\
U_{Y}\left(M_{2}^{\prime}, P\right)=U_{Y^{T}}\left(M_{2}, P^{\prime T}\right)^{\prime T} .
\end{gathered}
$$

By Theorem 4.5 there exists $\tau \in E_{\mathcal{B}}^{\mathcal{A}}$ with

$$
\mu_{I}(\tau) \geq 2+\frac{\log 2}{\log (2 \Gamma)}
$$

where

$$
\Gamma=\max _{X, P, Y}\left\{\frac{1}{\sqrt{\operatorname{det}\left(X P W_{Y}(X, P)^{\prime}\right)}}\left\|W_{Y}(X, P)\right\|_{\infty}\right\} .
$$

Note that when $b \geq 2$, then the asymptotic irrationality exponent $\mu_{I}(\tau)$ is bounded for every $\tau \in E_{\mathcal{B}}^{\mathcal{A}}$ by Theorem 1.1. For example when $a=1$ and $b=2$, then $\Gamma=13$ and $2.21 \leq \mu_{I}(\tau) \leq 5.69$.

The set $E_{\mathcal{B}}^{\mathcal{A}}$ does not always contain very well approximable numbers: all the sets $E_{\mathcal{B}}^{\{1\}}$ consist of badly approximable numbers, which have asymptotic irrationality exponent 2. Also, for example $E_{\{2,4\}}^{\{2\}} \subseteq E_{\{1,2,4\}}^{\{1\}}$, which shows that the upper bound in Theorem 1.1 is not always optimal. 


\section{References}

[B] Y. Bugeaud, Diophantine approximation and Cantor sets, Math. Ann. 341 (2008), 677-684.

[HLMT] J. Hančl, K. Leppälä, T. Matala-aho and T. Törmä, On irrationality exponents of generalized continued fractions, J. Number Theory 151 (2015), 18-35.

[K] K. Kolden, Continued fractions and linear substitutions, Arch. Math. Naturvid. 50 (1949), 151-196.

[LSV] J. Levesley, C. Salp and S. L. Velani, On a problem of K. Mahler: Diophantine approximation and Cantor sets, Math. Ann. 338 (2007), 97-118.

[M] K. Mahler, Some suggestions for further research, Bull. Austral. Math. Soc. 29 (1984), 101-108.

[R] G. N. Raney, On continued fractions and finite automata, Math. Ann. 206 (1973), 265-283.

[S] J. Steuding, Diophantine Analysis, Discrete Math. Appl. (Boca Raton), Chapman \& Hall/CRC, 2005.

Kalle Leppälä

Department of Mathematics

Aarhus University

Ny Munkegade 118

DK-8000 Aarhus C, Denmark

Present address:

iPsych

Aarhus University

Bartholins Allé 6

DK-8000 Aarhus C, Denmark

E-mail: kalle.m.leppala@gmail.com
Topi Törmä Department of Mathematical Sciences University of Oulu P.O. Box 8000 FI-90014 University of Oulu, Finland E-mail: topi.torma@oulu.fi 
Abstract (will appear on the journal's web site only)

We study which asymptotic irrationality exponents are possible for numbers in generalized continued fraction Cantor sets

$$
E_{\mathcal{B}}^{\mathcal{A}}=\left\{\frac{a_{1}}{b_{1}+\frac{a_{2}}{b_{2}+\cdots}}: a_{n} \in \mathcal{A}, b_{n} \in \mathcal{B} \text { for all } n\right\},
$$

where $\mathcal{A}$ and $\mathcal{B}$ are some given finite sets of positive integers. We give sufficient conditions for $E_{\mathcal{B}}^{\mathcal{A}}$ to contain numbers for any possible asymptotic irrationality exponent and show that sets with this property can have arbitrarily small Hausdorff dimension. We also show that it is possible for $E_{\mathcal{B}}^{\mathcal{A}}$ to contain very well approximable numbers even though the asymptotic irrationality exponents of the numbers in $E_{\mathcal{B}}^{\mathcal{A}}$ are bounded. 